\title{
Original
}

\section{Subcellular Localization and Phosphorylation of PHAPI in Mouse Fibroblasts}

\author{
Satoko Yamashina, Tamio Hagiwara, Tsunamasa Watanabe, \\ Hisa ShImojima and Minoru TAKedA
}

\begin{abstract}
The putative HLA-DR associated protein I (PHAPI) is a nuclear protein with a molecular mass of $32 \mathrm{kDa}$, which consists of about 250 amino acids. The cDNAs encoding the wild-type and mutated PHAPI were ligated into a eukaryotic expression vector, which produces the GFP fusion protein, and were expressed in the mouse fibroblast BALB3T3 cell line. The wild-type PHAPI and a series of mutated PHAPIs which lacked each 20-amino acid segment within the leucine-rich $\mathrm{N}$-terminal region were localized to nuclei, whereas a mutant protein which lacked the acidic C-terminal tail including the potential nuclear localization signal was diffusely spread throughout the whole cell. We have shown previously that PHAPI is phosphorylated in vitro by unknown protein kinases, at Ser-204 in human PHAPI. To investigate the phosphorylation of PHAPI in vivo, mutant PHAPI in which Ser-204 was changed to Ala was also expressed in the cells and was metabolically labeled with a radioactive orthophosphate. The mutant PHAPI was localized to nuclei in the same manner as wild-type PHAPI and was still phosphorylated at Ser residues although the phosphorylation level was slightly reduced compared to that of the wild-type PHAPI. These results suggest that PHAPI might be phosphorylated in vivo at several Ser residues including Ser-204, and that the phosphorylation of Ser-204 is not essential for the nuclear localization of PHAPI.
\end{abstract}

Key words : PHAPI, subcellular localization, phosphorylation

\section{Introduction}

Both PHAPI and PHAPII have been purified from the cytosolic fraction of the human lymphoblastoid B-cell line H2LCL using an affinity matrix composed of the synthetic biotinylated cytoplasmic region of the HLA-DR $\alpha$-chain immobilized on avidin agarose ${ }^{1)}$. The predominant structural feature of both PHAPI and PHAPII is a long stretch of acidic amino acids at the $\mathrm{C}$-terminal end. The $\mathrm{N}$-terminal regions of both proteins have no obvious sequence homology. Extended acidic regions are frequently found in proteins located in the nuclear compartment where they might interact with histones or other chromatin proteins $^{2,3)}$. Their primary structure suggests that both PHAPI and PHAPII may be involved in the generation of intracellular signaling events that lead to regulation of 
transcriptional activity after binding of a ligand to HLA class II molecules ${ }^{1)}$. Species of PHAPI have been found in a variety of biologic contexts, although the relationship to MHC function has not been precisely clarified. An essentially equivalent molecule, mapmodulin, was purified from $\mathrm{CHO}$ cells on the basis of its ability to stimulate the dynein- and microtuble-dependent localization of Golgi complexes ${ }^{4)}$. PHAPI has been variously identified as $\mathrm{LANP}^{5)}$, a leucine-rich nuclear protein from a neonatal rat cerebella, I1PP2A ${ }^{6}$, an inhibitor of protein phosphatase $2 \mathrm{~A}$ from bovine brain and $\mathrm{pp} 32^{7,8)}$, a tumor suppressor protein from human prostate cancer. We have also purified PHAPI from a cytosolic fraction of prenatal rat brain ${ }^{9)}$. These proteins are localized to different compartments of the cells such as the plasma membrane, cytoplasm and nucleus under certain circumstances.

We previously demonstrated that PHAPI is phosphorylated in vitro by unknown protein kinases that are partially purified from the cytosolic fraction of rat brain using a recombinant PHAPI protein-immobilized affinity matrix and that the phosphorylation site is Ser-204 residue in the human PHAPI ${ }^{10,11)}$. Interestingly, the phosphorylation site Ser-204 is located near the potent nuclear transport signal in the C-terminal region of PHAPI. In the present study, we investigated the phosphorylation and subcellular localization of PHAPI by expression of mutant PHAPI proteins in cultured cells.

\section{Experimental procedures}

Expression of cDNA sequences in mammalian cells

The cDNAs coding for the wild-type and mutant PHAPI were prepared as described previously ${ }^{10)}$. The pBluescript SK + vectors containing wild-type and mutant PHAPI cDNA were digested at the BamHI and PstI sites in the polylinker and the cDNA fragment was purified. These BamHI/PstI fragments containing PHAPI cDNA were ligated into the eukaryotic expression plasmid pEGFP-C1 (CLONTECH Laboratories, Inc., Palo Alto, CA, USA) which was cut with BamHI and PstI, and purified to remove the smaller fragment. These expression plasmids are expected to produce the green fluorescent protein (GFP)PHAPI fusion protein under the control of a cytomegalovirus promoter in eukaryotic cells. The mouse embryonic fibroblast BALB3T3 cell line was maintained in Dulbecco's modified Eagle's medium supplemented with $10 \%$ fetal bovine serum at $37^{\circ} \mathrm{C}$ in a humidified atmosphere of $5 \% \mathrm{CO}_{2}$ and $95 \%$ air. The recombinant expression plasmids were transfected into $2.5 \times 10^{5}$ BALB3T3 cells cultured in $60-\mathrm{mm}$ diameter dishes using LipofectAmine Plus reagent (GIBCO Life Technology, Inc., Rockville, MD, USA) according to the manufacturer's recommendation. At $48 \mathrm{hrs}$ posttransfection, cells were observed using a fluorescence microscope and were metabolically labeled with $\left[{ }^{32} \mathrm{p}\right]$ orthophosphate.

\section{Metabolic labeling and immunoprecipitation}

After $48 \mathrm{hrs}$ posttransfection, BALB3T3 cells were washed twice with phosphate-free minimal essential medium and then labeled for $2 \mathrm{hrs}$ at $37^{\circ} \mathrm{C}$ in $0.5 \mathrm{ml}$ of phosphate-free minimal essential medium with $24.7 \mathrm{MBq} / \mathrm{ml}$ of $\left[{ }^{32} \mathrm{p}\right]$ orthophosphate. The medium was then removed and the cells were washed with phosphate-buffered saline. The labeled cells were lyzed in $1 \mathrm{ml}$ of lysis buffer $(50 \mathrm{mM}$ Tris- $\mathrm{HCl}, \mathrm{pH} 8.0,150 \mathrm{mM} \mathrm{NaCl}, 1 \%$ Nonidet P-40, $0.25 \mathrm{mM}$ PMSF, $50 \mathrm{mM} \mathrm{NaF}, 2 \mathrm{mM}$ sorlium orthovanadate, $5 \mu \mathrm{M}$ okadaic acid, 2 mM EDTA, $2 \mathrm{mM}$ EGTA, $1 \mu \mathrm{g} / \mathrm{ml}$ antipain, $1 \mu \mathrm{g} / \mathrm{ml}$ leupeptin, $1 \mu \mathrm{g} / \mathrm{ml}$ pepstatin A) at 
$4^{\circ} \mathrm{C}$ for $1 \mathrm{hr}$. The lysate was centrifuged at $15,000 \mathrm{~g}$ for $10 \mathrm{~min}$ and then the supernatant was collected. The radiolabeled PHAPI proteins were immunoprecipitated by incubation with anti-GFP antibody (CLONTECH Laboratories, Inc.) followed by protein G-Sepharose beads (Amersham Pharmacia Biotech Inc., Upsala, Sweeden). The immunocomplexes were collected by centrifugation at $10,000 \mathrm{~g}$ for $15 \mathrm{sec}$ and then washed three times with $1 \mathrm{ml}$ of the lysis buffer. The beads were incubated in $25 \mu 1$ of Laemmli's sample buffer at $85^{\circ} \mathrm{C}$ for $10 \mathrm{~min}$ and subjected to SDS-polyacrylamide gel electrophoresis. The gel was dried and subjected to autoradiography.

\section{Phosphoamino acid analysis}

Phosphoamino acid analysis was performed as previously reported ${ }^{11)}$. In brief, the radiolabeled PHAPI protein was extracted from the gel and was hydrolyzed at $110^{\circ} \mathrm{C}$ for 1.5 hours in $6 \mathrm{~N} \mathrm{HCl}$. The hydrolysate was separated on cellulose thin layer by high-voltage electrophoresis. The radioactive amino acid was determined by comparison of the positions of the standard phosphoamino acids.

\section{Results}

\section{Subcellular localization of wild-type and mutated PHAPI proteins}

We previously constructed a series of mutant human PHAPI clones which lacked 20-amino acid segments of the coding region, and a mutant human PHAPI cDNA in which Ser-204 was changed to Ala. Fig. 1 shows a schematic comparison of these mutant clones. Of 12 serine residues of human wild-type PHAPI, one or two serine residues were missing in each deletion mutant. To investigate the subcellular localization of wild-type and mutant PHAPI proteins, expression plasmid in which the PHAPI cDNA was fused to the GFP gene was transfected in mouse BALB3T3 cells and the transiently expressed fluorescent fusion protein was observed (Fig. 2). The control GFP was diffusely expressed in the cytoplasm and nuclei (Fig. 2A). In contrast, the wild-type PHAPI was predominantly localized in the nuclei (Fig. 2B). A series of mutant proteins (DM1-DM7) which lacked each 20-amino acid segment of the coding region were also localized in the nuclei (Fig. 2C-I), however, the 168TAG mutant protein which lacked the acidic tail of the C-terminal region was diffusely distributed in the cytoplasm and nuclei (Fig. 2, J). There was no significant change in localization of fluorescent protein in which Ser-204 was changed to Ala (Fig. 2, K).

\section{Phosphorylation of PHAPI protein in BALB3T3 cells}

To confirm whether Ser-204 of human PHAPI is phosphorylated in cells, cells transiently expressing wild-type and mutant PHAPI were metabolically labelled with a radioactive phosphate. The GFP-PHAPI fusion protein was isolated by immunoprecipitation using a specific antibody against GFP. The native GFP as control and wild-type and mutant GFP-PHAPI fusion protein were the predicted sizes of 32 and $67 \mathrm{kDa}$ (Fig. 3, A). The native GFP itself was not phosphorylated in cells (Fig. 3B, lane 1). In contrast, both wild-type and mutant GFP-PHAPI proteins were phosphorylated, however, ${ }^{32} \mathrm{P}$-incorporation of the mutant GFP-PHAPI was slightly lower than that of the wild-type GFP-PHAPI, although the amounts of immunoprecipitated proteins were apparently equivalent (Fig. 3B, lanes 2 and 3$)$. The phosphoamino acid analysis revealed that the phosphorylation site(s) 


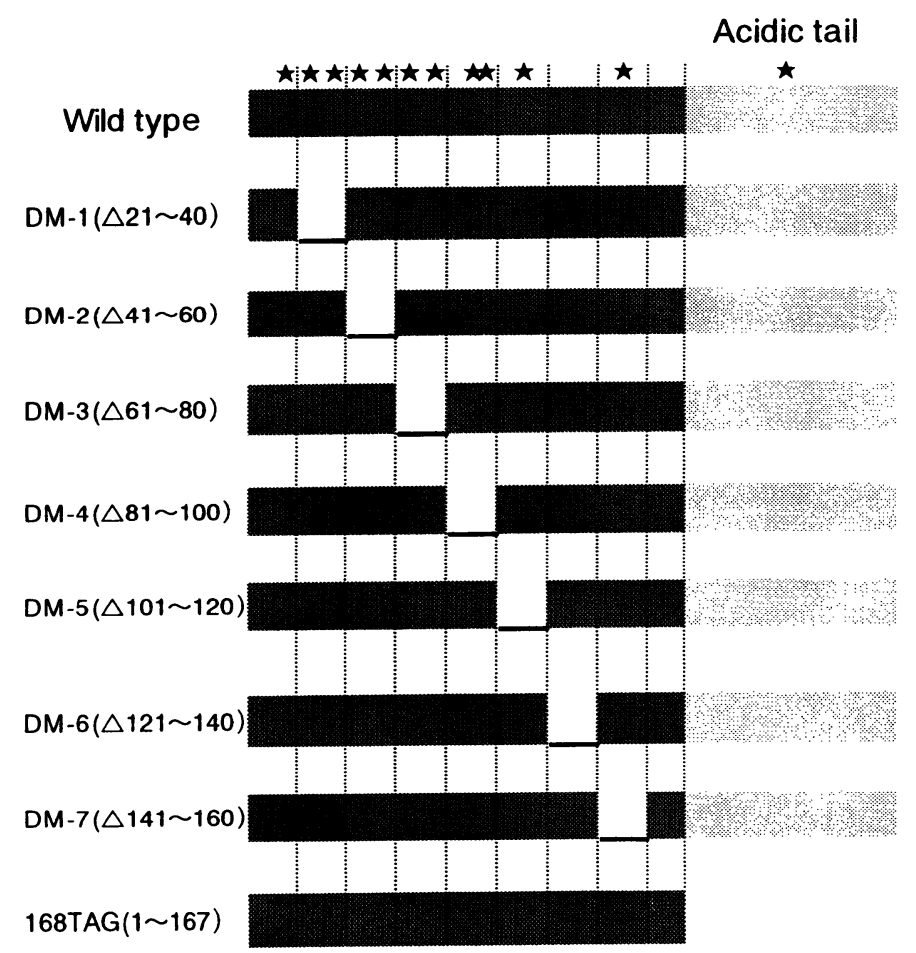

Fig. 1. Schematic structures of wild-type and mutant PHAPI

The wild-type human PHAPI consists of 249 amino acids including 12 serine residues. The stars indicate the positions of Ser-residues. The closed box and open box indicate the leucine-rich $\mathrm{N}$-terminal region and the acidic C-terminal tail, respectively. The mutant PHAPIs designated DM-1-DM-7 lacked sequential 20-amino acid segments in the leucine-rich $\mathrm{N}$-terminal region. The mutant PHAPI designated 168TAG had a truncated acidic C-terminal region.

of PHAPI was serine residue(s) but not threonine and tyrosine residues (Fig. 4).

\section{Discussion}

PHAPI species have been isolated from various species and tissues ${ }^{1,4-9)}$. The predominant structural features of PHAPI are the leucine-rich repeats in the $\mathrm{N}$-terminal region and the cluster of acidic amino acids in the $\mathrm{C}$-terminal region. Leucine-rich repeats are found in many eukaryotic proteins and potentially function in protein-protein interactions. The cluster of acidic amino acids in the C-terminal region of PHAPI includes a short stretch of basic amino acids, Lys-Arg-Lys-Arg, which agrees with the consensus sequence, Lys-Arg/L ys-X-Arg/Lys for nuclear targeting ${ }^{12)}$. PHAPI and homologues such as pp32, LANP and mapmodulin have been found in a variety of subcellular compartments. Immunohistochemical studies have indicated that LANP and pp32 are localized in nuclei of Perkinje cells in rat cerebellum and human prostatic neoplasia, respectively ${ }^{5,7)}$. PHAPI and mapmodulin were purified from the cytosolic fractions of cultured cell lines and rat brain ${ }^{1,4-9)}$. These different localizations of PHAPI are thought to be due to differences in the experimental conditions. We previously demonstrated that Ser-204 of human PHAPI protein is 

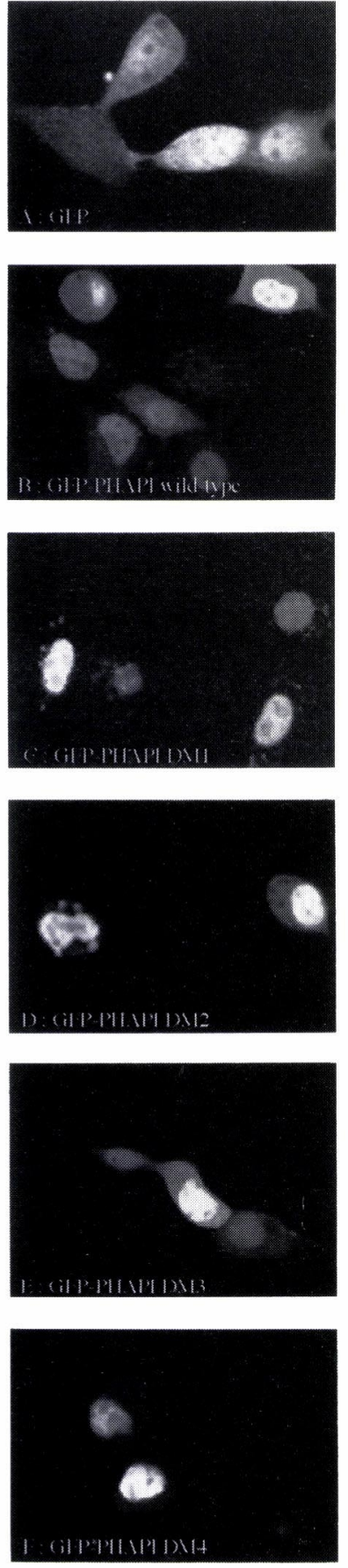

Fig. 2. Subcellular localization of wild-type and mutant PHAPI Mouse BALB3T3 cells were transiently transfected with either wild-type, mutant PHAPI or control vector and observed by confocal laser scanning microscope after $48 \mathrm{hrs}$ posttransfection. 


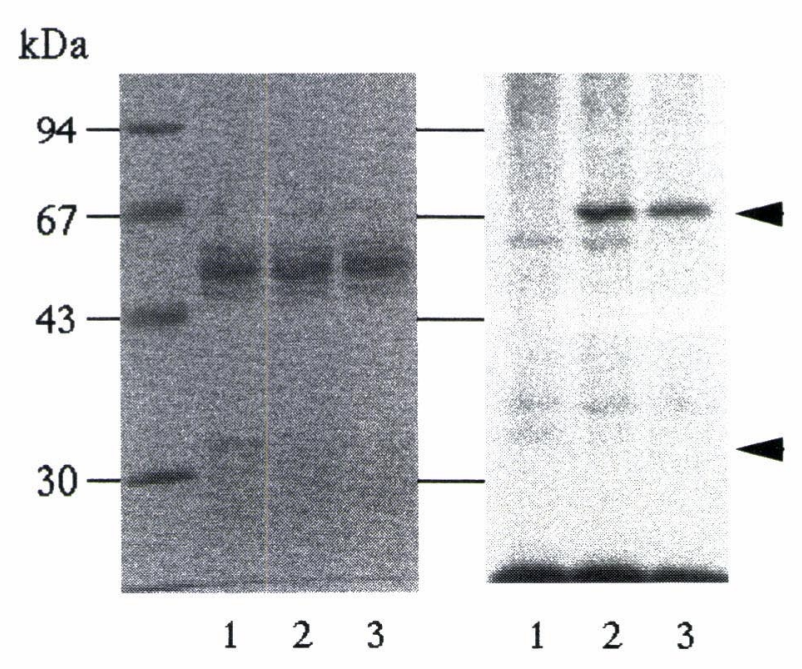

Fig. 3. Immunoprecipitation of GFP-PHAPI fusion protein labeled metabolically in BALB3T3 cells

Mouse BALB3T3 cells were transiently transfected with either wild-type, mutant PHAPI (S204A) or control vector and were metabolically labeled with a radioactive orthophosphate $48 \mathrm{hrs}$ posttransfection. Cells were lyzed and then GFP or GFP-PHAPI fusion protein were immunoprecipitated using a specific antibody against GFP. The immunocomplex was separated by SDS-PAGE and the gel was subjected to autoradiography. The left panel shows a CBB-staining gel and the right panel was an autoradiogram $f$ the same gel. Lane 1; GFP alone (control vector), lane 2; GFP-wild-type PHAPI, lane 3; GFP-S204A PHAPI. Arrowheads indicate the positions of GFP and GFP-PHAPI fusion proteins.

phosphorylated in vitro by unknown protein kinases which are partially purified from the cytosolic fraction of rat brain ${ }^{10,11)}$. Interestingly, Ser-204 is located near the putative nuclear localization signal within the cluster of acidic amino acids. Therefore, we speculated that the phosphorylation of Ser-204 of PHAPI might regulate the nuclear export/import of PHAPI.

In this study, we investigated the phosphorylation and subcellular localization of mutant PHAPI proteins generated by a site-directed mutagenesis. As expected, the transiently expressed PHAPI was phosphorylated at the Ser-residue in the BALB3T3 cells. However mutant PHAPI in which the putative phosphorylation site, Ser-204 was changed to Ala was also phosphorylated at the Ser-residue although the phosphate-incorporation of mutant PHAPI was reduced compared to that of wild-type PHAPI, suggesting that PHAPI might be phosphorylated at Ser-204 and at additional Ser-residue(s) in cells. Several investigators have suggested that PHAPI contains potential motifs for phosphorylation by protein kinases such as protein kinase $\mathrm{C}$, protein kinase $\mathrm{A}$, casein kinases and tyrosine protein kinases ${ }^{1,13)}$. However, in BALB3T3 cells, there was no detectable phosphorylation of Thr- and Tyr-residues of PHAPI (Fig. 3).

The wild-type PHAPI was localized mainly to nuclei, while mutant PHAPI which lacked the cluster of acidic amino acids in the C-terminal tail was diffusely expressed throughout the whole cell. These results confirmed that the nuclear translocation of PHAPI is 


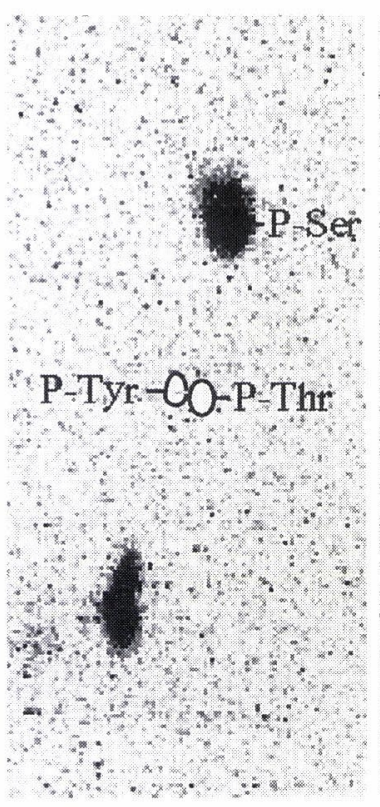

A

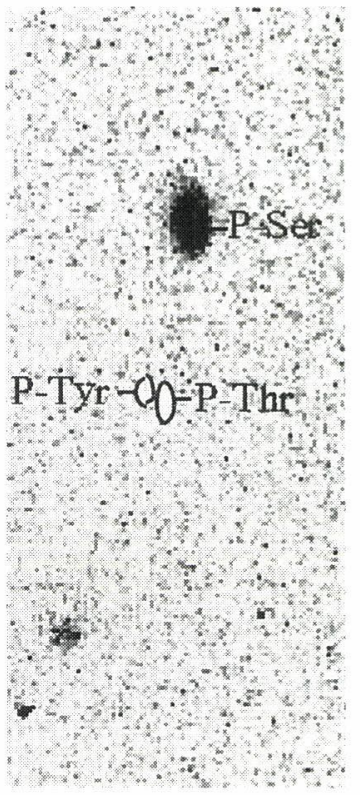

B

Fig. 4. Phosphoamino acid analysis

The protein bands of metabolically labeled GFP-PHAPI (shown in

Fig. 3) were excised from the gel and were subjected to phosphoamino acid analysis as described in "Experimental procedures".

A ; GFP-wild-type PHAPI, B ; GFP-S204A PHAPI.

regulated by the short stretch of basic amino acids, Lys-Arg-Lys-Arg within the cluster of acidic amino acids in the C-terminal tail. However, the substitution of Ser-204 to Ala of PHAPI had no effect on the subcellular localization of PHAPI. At present it is unknown whether the phosphorylation of PHAPI may regulate functions other than nuclear localization or whether unphosphorylated PHAPI may still be translocated to the nuclei.

PHAPI is implicated in diverse cellular functions such as MHC class II signaling, tumorigenesis and cytoskeletal regulation. Although the regulation of the functional activities of PHAPI is unclear, the phosphorylation and translocation of PHAPI might be involved in multiple PHAPI functions.

\section{References}

1) Vaesen M, Barnikol-Watanabe S, Gotz H, Awni LA, Cole T, Zimmermann B, Kratzin HD and Hilschmann $\mathrm{N}$ : Purification and characterization of two putative HLA class II associated proteins: PHAPI and PHAPII. Biol Chem Hoppe-Seyler 375 : 113-126 (1994)

2) Kuehl L, Childers TJ and McCauley RM: The occurrence of extended acidic sequences in nonhistone chromosomal proteins. Arch Biochem Biophys 248 : 272-281 (1986)

3) Earnshaw WC: Anionic regions in nuclear proteins. J Cell Biol 105 : 1479-1482 (1987)

4) Ulitzer N, Rancano C and Pfeffer SR: Biochemical characterization of mapmodulin, a protein that binds microtuble-associated proteins. J Biol Chem 272 : 30577-30582 (1997)

5) Matsuoka K, Taoka M, Satozawa N, Nakayama H, Ichimura T, Takahashi N, Yamakuni T, Song SY and Isobe T: A nuclear factor containing the leucine-rich repeats expressed in murine cerebellar neurons. Proc Natl Acad Sci USA 91 : 9670-9674 (1994) 
6) Li M, Makkinje A and Damuni Z: Molecular identification of I1PP2A, a novel potent heat-stable inhibitor protein of protein phosphatase 2A. Biochemistry $35: 6998-7002$ (1996)

7) Walensky LD, Coffey DS, Chen TH, Wu TC and Pasternack GR: A novel Mr 32,000 nuclear phosphoprotein is selectively expressed in cells competent for selfrenewal. Cancer Res 53 : 4720-4726 (1993)

8) Kadkol SS, Brody JR, Pevsner J, Bai J and Pasternack GR : Modulation of oncogenic potential by alternative gene use in human prostate cancer. Nature Med 5 : 275-279 (1999)

9) Sato T, Hagiwara T, Aoki K, Kuraishi H, Nishigaki Y, Tateno K, Tanaka T, Takeda F and Takeda M: A novel PHAPI-related 35-kD protein highly expressed in the developing brain. Showa Univ J Med Sci 11 : 59-67 (1999)

10) Tateno K, Hagiwara T, Tanaka $T$, Motohashi $K$, Takeda $\mathbf{F}$ and Takeda $\mathbf{M}$ : Investigation for target protein of putative human leukocyte antigen-DR associated protein I (PHAP I) and II (PHAP II). J Showa Med Assoc 59 : 195-201 (1999) (in Japanese)

11) Tanaka T, Hagiwara T, Tateno $K$, Motohashi $K$, Takeda $F$ and Takeda $M$ : Identification of PHAP I phosphorylated site. J Showa Med Assoc 59 : 202-207 (1999) (in Japanese)

12) Chelsky $D$, Ralph $R$ and Jonak $G$ : Sequence requirements for synthetic peptidemediated translocation of the nucleus. Mol Cell Biol 9 : 2487-2492 (1989)

13) Malek SN, Katumuluwa AI and Pasternack GR : Identification and preliminary characterization of two related proliferation-associated nuclear phosphoproteins. J Biol Chem 265 : 13400-13409 (1990)

[Received November 21, 2000 : Accepted December 13, 2000] 